Hitoshi Mikami • Masahito Ogasawara • Yoichi Matsubara Masahiro Kikuchi • Shigeaki Miyabayashi • Shigeo Kure Kuniaki Narisawa

\title{
Molecular analysis of methylmalonyl-CoA mutase deficiency: identification of three missense mutations in mut $^{0}$ patients*
}

Received: July 28, 1998 / Accepted: September 4, 1998

\begin{abstract}
Genetic defects in the methylmalonyl-CoA mutase (MCM) gene cause methylmalonic acidemia (MMA). Only three mutations have been reported among Oriental patients to date. We studied fibroblast cell lines established from three Japanese patients with MCM deficiency. Enzymatic study showed that these patients had the $m u t^{0}$ type of MMA. Nucleotide sequencing of MCM cDNAs identified three missense mutations: a $\mathrm{T}$ to $\mathrm{A}$ change at nucleotide position 2082, which results in an amino acid substitution of Glu669 for valine (V669E); a T to A change at position 1179 with the corresponding amino acid substitution of Asp368 for valine (V368D); and a $\mathrm{G}$ to A change at position 1182 with the corresponding amino acid substitution of His369 for arginine $(\mathrm{R} 369 \mathrm{H})$. Each of the three missense mutations abolished MCM activity according to a transient expression study. Alignment of these mutations with a recently reported homology model of human MCM allowed us to speculate on the effect of these nonconservative amino acid substitutions on MCM activity: V368D and R369H affected residues in the $\beta / \alpha$-(TIM-) barrel domain, on one of the two $\alpha$-helices that form the dimer interface, while V669E altered a residue in the adenosylcobalamin-binding domain in the $\mathrm{C}$ terminus.
\end{abstract}

Key words Methylmalonic acidemia $\cdot$ Methylmalonyl-CoA mutase $\cdot$ Adenosylcobalamin $\cdot$ Organic aciduria $\cdot$ Mutation

H. Mikami · M. Ogasawara · Y. Matsubara $(\bowtie) \cdot$ S. Kure

K. Narisawa

Department of Medical Genetics, Tohoku University School of

Medicine, 1-1 Seiryomachi, Aoba-ku, Sendai 980-8574, Japan

Tel. +81-22-717-8138; Fax +81-22-717-8142

e-mail: ymats@mail.cc.tohoku.ac.jp

M. Kikuchi · S. Miyabayashi

Department of Pediatrics, Tohoku University School of Medicine, Sendai, Japan

*A portion of this work was presented at the 44th Annual Meeting of the American Society of Human Genetics (Ogasawara et al. 1994a).

\section{Introduction}

Methylmalonyl-CoA mutase (MCM, EC 5.4.99.2) deficiency is an autosomal recessive disorder characterized clinically by neonatal or infantile metabolic ketoacidosis (Fenton and Rosenberg 1995). It is responsible for mut types of methylmalonic aciduria (MMA), whereas $c b l$ types of MMA are due to inherited abnormalities in the metabolism of adenosylcobalamin, a cofactor for MCM. The mut types of MMA are further classified into two subtypes: $m u t^{-}$types exhibit decreased MCM activity with an altered $K_{\mathrm{m}}$ for adenosylcobalamin; $m u t^{0}$ types show no residual activity even in the presence of excess cofactor (Fenton and Rosenberg 1995). MCM is a homodimer of identical subunits with an apparent molecular weight of $77.5 \mathrm{kDa}$ and is located in the mitochondrial matrix (Fenton et al. 1982). The MCM gene has been mapped to human chromosome 6p12-21.1, consists of 13 exons, spreads over more than $30 \mathrm{~kb}$, and produces mRNA with a length of $2.7 \mathrm{~kb}$ (Ledley et al. 1988; Jansen et al. 1989; Nham et al. 1990).

Molecular analysis of MCM deficiency among Caucasian and African-American patients has revealed at least 20 mutations to date (Ledley and Rosenblatt 1997; Adjalla et al. 1998). Each of the mutations was found only in one family, except for G717X, which has been identified in three patients of African-American origin evaluated at one hospital (Crane et al. 1992), suggesting considerable genetic heterogeneity of MCM deficiency in Caucasian populations. Only three mutations have been reported in Oriental patients. We previously reported a nonsense mutation (g425t, which results in E117X) and a two-base deletion frame shift (769delCA) in Japanese patients (Ogasawara et al. 1994b). A splicing error mutation (INV2 $+5 \mathrm{G}>\mathrm{A}$ ) was also described by Toyo-Oka et al. (1995). The E117X substitution accounted for 7 of 32 mutant MCM alleles (22\%), while each of the other two mutations were found in a single family. The presence of a prevalent E117X mutation and the absence of a missense mutation suggested that the spectrum of MCM mutations in Oriental patients may be 
distinct from that of Caucasians. However, the majority of MCM mutations in Oriental populations remain to be elucidated. We report here on the identification of three missense mutations responsible for $m u t^{0}$ MMA. Alignment of these mutations with a recently reported homology model of human MCM allows us to speculate on the effect of each substitution on MCM activity (Thomä and Leadlay 1996).

\section{Materials and methods}

\section{Patients}

We studied three Japanese patients with MCM deficiency who showed initial symptoms, such as vomiting, poor feeding, and/or respiratory distress, during the neonatal period (days 1-8). Although they survived the acute phase with intensive medical treatment, moderate to severe mental retardation was observed in all patients.

\section{Enzymatic analysis of fibroblast cultures}

Fibroblast cultures (Nos. 69, 133, and 422) were obtained from the three patients. The diagnosis was enzymatically confirmed by our previously described method employing high-performance liquid chromatography (Kikuchi et al. 1989). A propionate incorporation study was also carried out to distinguish between the $m u t^{-}$and $m u t^{0}$ types (Willard et al. 1976).

\section{Extraction of RNA and Northern blotting}

mRNA was isolated from cultured skin fibroblasts according to the acid guanidinum thiocyanate-phenol-chloroform extraction method (Chomczynski and Sacchi 1987) followed by oligo-dT column (Pharmacia LKB Biotechnology, Uppsala, Sweden) chromatography. One microgram of mRNA was electrophoresed through a $1.0 \%$ agarose gel in the presence of formaldehyde and transferred to a nylon membrane (Sambrook et al. 1989). The membrane was hybridized with ${ }^{32} \mathrm{P}$-labeled MCM cDNA, and subsequently with ${ }^{32} \mathrm{P}$-labeled $\beta$-actin cDNA.

Reverse transcription/polymerase chain reaction (PCR) of MCM mRNA and nucleotide sequencing of MCM cDNA

One microgram of the isolated poly $(\mathrm{A}+) \mathrm{RNA}$ was used to synthesize first-strand cDNA, and the entire coding region of MCM cDNA was amplified by PCR as described previously except that each amplification primer had an additional BamHI or StuI recognition sequence at the $5^{\prime}$ end (Ogasawara et al. 1994b). PCR products were digested with BamHI and StuI (Takara, Kyoto, Japan) and subcloned into the BamHI-SmaI site of pGEM-Blue plasmid (Promega, Madison WI, USA). Isolated plasmid from multiple inde- pendent clones was subjected to sequencing analysis by the dideoxy chain termination method.

\section{Direct sequencing of MCM cDNA and genomic} MCM locus

To confirm the presence of mutations detected by the plasmid-sequencing analysis and to identify allelic status of the mutations, PCR copies of MCM cDNA and genomic DNA were sequenced directly (Winship 1989). Primer sequences in introns were based on data on the human genomic MCM structure (Ogasawara et al., unpublished data). The direct sequencing was performed by the following protocol. Amplified fragments of MCM cDNA and genomic MCM DNA were electrophoresed on a $1 \%$ agarose gel, visualized with ethidium bromide. The desired bands were excised from the gel and the DNA was recovered by a glass powder method. The purified templates $(0.5 \mathrm{pmol})$ were mixed with primers $(100 \mathrm{pmol})$ in $40 \mathrm{mM}$ Tris $\mathrm{HCl}(\mathrm{pH}$ 7.5), $20 \mathrm{mM} \mathrm{MgCl}_{2}, 50 \mathrm{mM} \mathrm{NaCl}$, and $10 \%$ dimethyl sulfoxide. The mixture was boiled for $3 \mathrm{~min}$ and immediately immersed in liquid nitrogen. The template-primer mixture was then placed on ice and subjected to sequencing reaction using Sequenase (USB, Cleveland $\mathrm{OH}, \mathrm{USA}$ ) according to the manufacturer's protocol except that $10 \%$ dimethyl sulfoxide was included throughout the reaction.

Detection of g425t mutation by $M b o$ II restriction enzyme digestion

The E117X mutation, which has been found in Japanese patients with a relatively high incidence, was screened by a previously described method by PCR amplification of a DNA fragment in exon 2/intron 2 followed by digestion with MboII (New England Biolabs, Beverly, MA, USA).

Transient expression study

MCM-deficient fibroblast cell culture 325 was transformed by replication origin-defective simian virus 40 ( ori $^{-}$SV40) and used for transient expression analysis of various MCM mutations (Gulzman et al. 1980).

MCM mutations were introduced into MCM cDNA by a Transformer site-directed mutagenesis kit (Clontech, Palo Alto, CA, USA) according to the manufacturer's protocol. Normal or mutant MCM cDNA was subcloned into a mammalian expression vector, pUC-CAGGS (Niwa et al. 1991), which carries chicken $\beta$-actin promoter and cytomegalovirus enhancer. The sequence of MCM cDNA in the recombinant expression vector was confirmed by nucleotide-sequencing analysis. Culture 325 with ori ${ }^{-}$SV40 seeded in a $175 \mathrm{~cm}^{2}$ flask was transfected with each of the recombinant vectors $(20 \mu \mathrm{g})$ and pSV- $\beta$-gal $(5 \mu \mathrm{g})$ using Lipofectamine reagent (Gibco BRL, Gaithersburg MD, USA). The cells were harvested $72 \mathrm{~h}$ after the transfection and assayed for MCM activity and $\beta$-galactosidase activity. 


\section{Results}

MCM activity was undetectable (less than 100 pmol of succinyl-CoA formed per minute per milligram protein, while the control value in normal fibroblasts was $2019 \pm$ 434 pmol of succinyl-CoA formed per minute per milligram protein) in all three fibroblast cultures derived from the patients with or without the presence of excess adenosylcobalamin $(5 \mu \mathrm{M})$ by the high-performance liquid chromatographic method. The more sensitive propionate incorporation study showed that propionate incorporation was not stimulated by a high concentration of hydroxycobalamin $(1 \mu \mathrm{g} / \mathrm{ml})$ in the cultures, indicating that these cells exhibited a $m u t^{0}$ phenotype.

Screening of genomic DNA for the E117X mutation, which was associated with markledly decreased mRNA (Ogasawara et al. 1994b), revealed that both cultures 133 and 422 were heterozygous for the mutation. Northern blot analysis detected MCM mRNA in the three cultures (Fig. 1, lanes 2, 3, and 4). We then analyzed MCM mRNA from the three cultures by reverse transcription/PCR amplification of MCM cDNA followed by nucleotide sequencing. The obtained nucleotide sequences were compared with human MCM cDNA sequence in the GenBank database (accession Nos. M37510 and J04774), since the original report of MCM cDNA contained several cloning or sequencing errors (Jansen et al. 1989). The most significant difference was a deletion of 24 nucleotides (nt 1382-1405) in the previous report. The nucleotides were in-frame, adding an extra eight amino acids (NDVYDAAL), and did not alter the downstream amino acid sequences. Our sequencing analy-
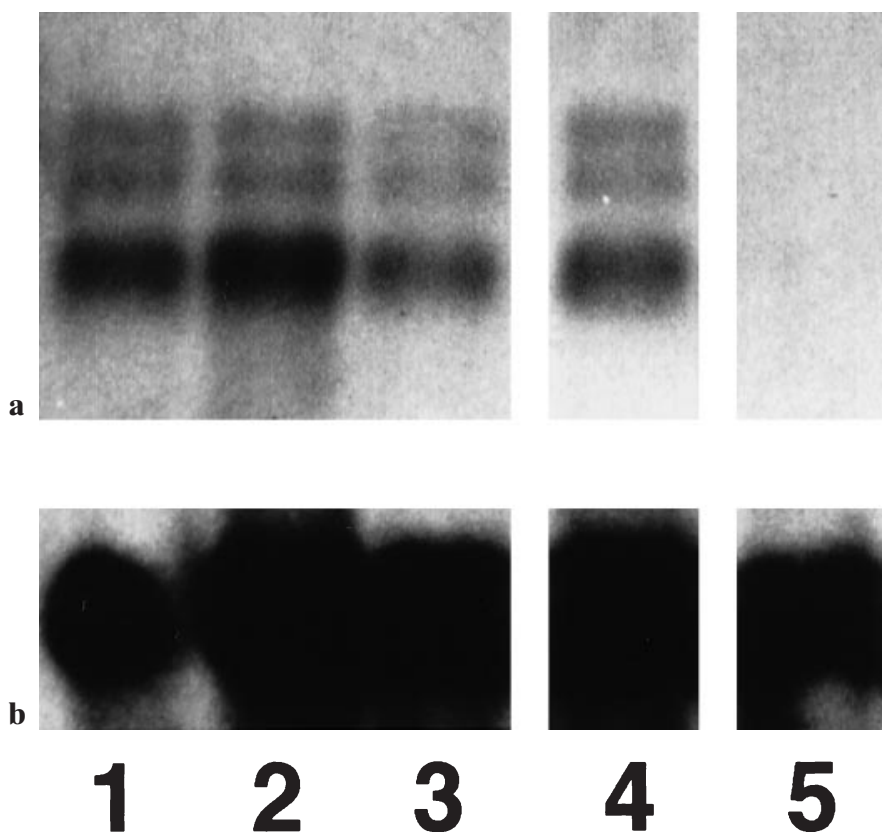

Fig. 1 Northern blot analysis. Lane 1, control fibroblast; lane 2, fibroblast culture 69; lane 3, fibroblast culture 133; lane 4, fibroblast culture 422; lane 5, fibroblast culture 325 . The membrane was hybridized with $\mathbf{a}$ an MCM cDNA probe and $\mathbf{b}$ subsequently with a $\beta$-actin cDNA probe sis of MCM cDNA from two normal Japanese individuals indicated that the 24 nucleotides were indeed present in normal cDNA, and the subunit of MCM precursor protein consists of 750 amino acids. Other differences were singleor double-nucleotide changes.

Sequencing of MCM cDNA obtained from culture 69 revealed a single-base change of $\mathrm{T}$ to $\mathrm{A}$ at nucleotide position 2082 in MCM cDNA, which resulted in an amino acid substitution of Glu669 for Val669 (V669E) in exon 12 (Fig. 2a). Direct sequencing of amplified MCM cDNA identified the same single-base substitution. Exon 12 of the MCM gene was then amplified with intronic primers (sense primer, 5'-GGTATTGCTAGATTGTCTTCC-3'; antisense primer, 5'-TCAAGATTCCCAATCACAGTAC- $3^{\prime}$ ) and directly sequenced. The sequencing analysis showed the presence of both $\mathrm{T}$ and $\mathrm{A}$ at this position, indicating that the cell line was a compound heterozygte of the V669E mutation and another as yet unidentified mutation associated with markedly reduced expression.

Sequencing of MCM cNDA obtained from culture 133 identified a single-base change of $\mathrm{T}$ to $\mathrm{A}$ at nucleotide position 1179, which resulted in an amino acid substitution of Asp368 for Val368 (V368D) in exon 6 (Fig. 2b). Direct sequencing of genomic DNA indicated that culture 133 is a compound heterozygote of V368D and E117X.

Sequencing of MCM cDNA obtained from culture 422 revealed a single-base change of $\mathrm{G}$ to $\mathrm{A}$ at position 1182 , which resulted in an amino acid substitution of His369 for Arg369 (R369H) in exon 6 (Fig. 2c). Direct sequencing of genomic DNA showed that culture 422 is a compound heterozygote of R369H and E117X.

Transient expression studies were carried out to determine whether the identified missense mutations were causes of MMA in these patients. We used fibroblast culture 359 for the transfection with each recombinant expression vector instead of COS cells, because COS cells exhibited a significantly high level of basal MCM activity. Culture 359 was first transformed with ori ${ }^{-}$SV40 to obtain an enhanced growth rate and an unlimited life span. The transformed culture did not show MCM activity (Table 1). Normal and mutated MCM cDNA was introduced into the culture and transiently expressed under the control of

Table 1 Transient expression analysis of MCM cDNA harboring missense mutations identified in the study. Each transfection experiment was performed in triplicate. The efficiency of transfection was monitored by assaying $\beta$-galactosidase activity in the cell extract

\begin{tabular}{|c|c|}
\hline Expression vector used for transformation & $\mathrm{MCM}_{\text {activity }}{ }^{\mathrm{a}}$ \\
\hline Mock & $\mathrm{ND}^{\mathrm{b}}$ \\
\hline pUC-CAGGS/normal MCM cDNA (sense) & $1508 \pm 442$ \\
\hline pUC-CAGGS/normal MCM cDNA (antisense) & ND \\
\hline pUC-CAGGS/MCM cDNA with V368D & ND \\
\hline pUC-CAGGS/MCM cDNA with R369H & ND \\
\hline pUC-CAGGS/MCM cDNA with V669E & ND \\
\hline Normal fibroblast cultures $(n=8)$ & $2019 \pm 434$ \\
\hline
\end{tabular}

${ }^{\mathrm{a}}$ Expressed as picomoles of succinyl-CoA formed per minute per milligram protein

${ }^{\mathrm{b}}$ Not detected (less than $100 \mathrm{pmol}$ of succinyl-CoA formed per minute per milligram protein) 


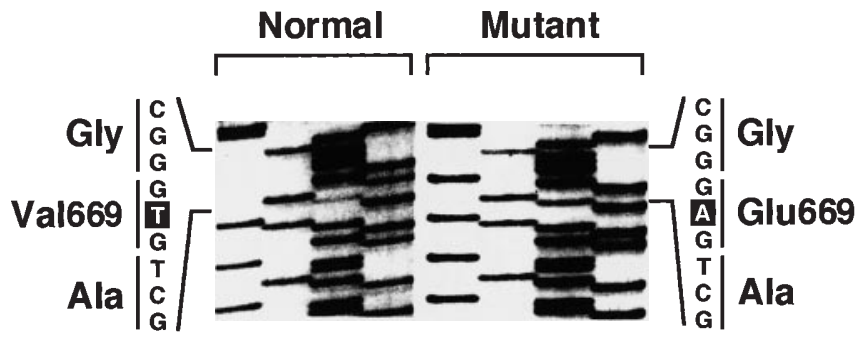

a $\begin{array}{llllllll}A & C & G & T & A & C & G & T\end{array}$
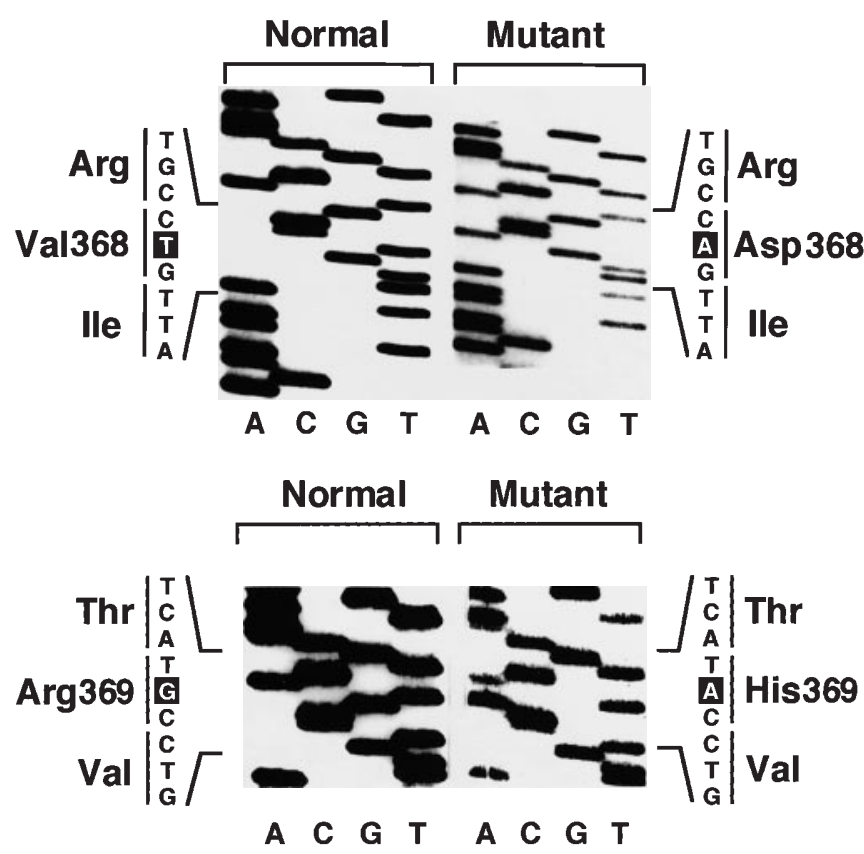

Fig. 2 a Nucleotide sequencing of MCM cDNA derived from culture 69. The autoradiogram shows a base substitution of $\mathrm{T}$ to $\mathrm{A}$, which resulted in an amino acid substitution of Glu669 for valine. b Nucleotide sequencing of MCM cDNA derived from culture 133. The autoradiogram shows a base substitution of $\mathrm{T}$ to $\mathrm{A}$, which resulted in an amino acid substitution of Asp368 for valine. c Nucleotide sequencing of MCM cDNA derived from culture 422. A substitution of $G$ to $A$, which resulted in an amino acid substitution of His369 for arginine was identified

chicken $\beta$-actin promoter and cytomegalovirus enhancer. Normal MCM cDNA in pUC-CAGGS increased the MCM specific activity from undetectable to $1508 \pm 442 \mathrm{pmol}$ of succinyl-CoA formed per minute per milligram protein, which was comparable to the value in normal fibroblasts (Table 1). In contrast, increase of MCM activity was not observed by the transfection of any of the expression vectors carrying the three different point mutations, V669E, V368D, or R369H (Table 1).

\section{Discussion}

Recently, the crystal structure of MCM from Propionibacterium shermanii at $2 \AA$ resolution was reported, and the cobalamin-binding and coenzyme substrate-binding domains were identified (Mancia et al. 1996). Furthermore a three-dimensional homology model of human MCM generated from the structure of the $\alpha$ subunit of $P$. shermanii MCM provided valuable information for interpreting the effect of various mutations on MCM function (Thomä and Leadlay 1996). The three mutations identified in this study substitute amino acid residues that are conserved between human MCM and the $\alpha$ subunit of $P$. shermanii MCM. Both V368D and R369H affect amino acid residues in helix I $\alpha 7$ of the $\beta / \alpha$-(TIM-) barrel domain on one of the two $\alpha$ helices that form the dimer interface. An arginine residue at 369 is salt bridged to Glu415 provided by the other subunit. Therefore, these two mutations might affect dimerization of MCM subunits, possibly destabilizing mutant MCM proteins.

$\mathrm{R} 369 \mathrm{H}$ has been independently described in a report by Adjalla et al. (1998) as "unpublished results" by Kogekar, Janata and Fenton. It was reported that the mutation was associated with low mRNA phenotype. However, our Northern blot analysis of cell culture 422, which is a compound heterozygote of $\mathrm{R} 369 \mathrm{H}$ and E117X, showed approximately the normal amount of MCM mRNA (Fig. 1, lane 4). Since E117X homozygotes exhibited MCM mRNA at levels less than 1\% of normal controls (Ogasawara et al. 1994b), it seems unlikely that $\mathrm{R} 369 \mathrm{H}$ is associated with decreased amounts of MCM mRNA.

V669E affected an amino acid residue in the adenosylcobalamin-binding domain in the $\mathrm{C}$ terminus of MCM, where clustering of MCM mutations has been noted (Crane and Ledley 1994). The core of the $B_{12}$ binding domain is formed by five parallel $\beta$ strands (II $\beta 1-$ II $\beta 5$ ) that alternate with $\alpha$ helices (II $\alpha 1-\mathrm{II} \alpha 5$ ), and a valine residue at 669 is located in strand II $\beta 3$ (Thomä and Leadlay 1996). Strands II $\beta 3$ and II $\beta 4$ form one side of a deeply buried pocket in which the dimethylbenzimidazole portion of the cobalamin is accommodated, while the other side of the pocket is flanked by side-chains from helices II $\alpha 1$ and II $\alpha 5$. The V669E mutation results in a replacement of a conserved hydrophobic residue with an acidic hydrophilic residue, and is likely to affect the key function of the enzyme. Also, there is a possibility that the mutation affects the stability of mutant MCM protein.

Our initial attempt of in vitro expression analysis of each mutation was performed in COS cells using an eukaryotic expression vector, pEUK-C1 (Clontech). However, a significantly high level of intrinsic MCM activity in COS cells and a relatively weak gene expression driven by the SV40 late promoter hampered precise measurement of extraneous MCM activity. To establish a cell culture suitable for the in vitro expression study of MCM, we transformed MCMdeficient fibroblast culture 359 with ori $^{-}$SV40. Since MCM mRNA in culture 325 was not detected by Northern blot analysis (Fig. 1, lane 5) and no mutase protein was detected by Western blot analysis using anti-MCM antibody (unpublished results), it is unlikely to have complementation with extrinsic mutant MCM protein expressed in the cells. Molecular analysis of culture 325 indicated that it is a compound heterozygote of E117X and another as yet unidentified mutation. The transformed and cloned culture 
originating from culture 325 did not show MCM activity. The establishment of the expression system allowed us to measure precisely the MCM activity produced by each mutant MCM cDNA. None of the three mutations exhibited residual MCM activity in our experiments. This observation is in line with the possible detrimental effect of each amino acid substitution on an important functional domain in the MCM protein, and is compatible with the severe clinical pictures of the three patients.

Our previous study showed the relatively high incidence of the E117X mutation among Japanese patients (Ogasawara et al. 1994b). In contrast, the 769 $\Delta$ ca, V669E, $\mathrm{V} 368 \mathrm{D}$, and $\mathrm{R} 369 \mathrm{H}$ mutations were identified only once in 18 patients with MCM deficiency (data not shown). These results suggested that MCM deficiency may also be genetically heterogeneous in Japanese populations. Since MCM deficiency is one of the most prevalent organic acidemias in Japan, it is important to further characterize MCM mutations to establish DNA diagnosis of MMA in the population. Also, the interpretation of various mutations with defined clinical pictures and biochemical properties in relation to three-dimensional homology modeling of human MCM would provide further insights into the genotypephenotype correlation of MCM deficiency and the structure-function relationship in the MCM protein.

Acknowledgments The authors are indebted to Dr. Fred D. Ledley for generously providing us with MCM cDNA at the initial stage of the study and Dr. Jun-ichi Miyazaki for a gift of pUC-CAGGS. This work was supported by the Grant-in-Aid for Scientific Research from the Ministry of Education, Culture, Sports and Science of Japan and grants from the Ministry of Health and Public Welfare of Japan.

\section{References}

Adjalla CE, Hosack AR, Gilfix BM, Lamothe E, Sun S, Chan A, Evans S, Matiaszuk NV, Rosenblatt DS (1998) Seven novel mutations in mut methylmalonic aciduria. Hum Mutat 11: 270-274

Chomczynski P, Sacchi N (1987) Single-step method of RNA isolation by acid guanidinum thiocyanate-phenol-chloroform extraction. Anal Biochem 162: 156-159

Crane AM, Ledley FD (1994) Clustering of mutations in methylmalonyl CoA mutase associated with mut ${ }^{-}$methylmalonic acidemia. Am J Hum Genet 55: 42-50

Crane AM, Martin LS, Valle D, Ledley FD (1992) Phenotype of disease in three patients with identical mutations in methylmalonyl CoA mutase. Hum Genet 89: 259-264
Fenton WA, Rosenberg LE (1995) Disorders of propionate and methylmalonate metabolism. In Scriver CR, Beaudet AL, Sly WS, Valle D (eds) The metabolic and molecular bases of inherited disease, 7th edn. McGraw-Hill, New York, pp 1423-1449

Fenton WA, Hack AM, Willard HF, Gertler A, Rosenberg LE (1982) Purification and properties of methylmalonyl coenzyme A mutase from human liver. Arch Biochem Biophys 214: 815-823

Gulzman Y, Frisque RJ, Sambrook J (1980) Origin-defective mutants of SV40. Cold Spring Harbor Symp Quant Biol 44: 293-300

Jansen R, Kalousek F, Fenton WA, Rosenberg LE, Ledley FD (1989) Cloning of full-length methylmalonyl-CoA mutase from a cDNA library using the polymerase chain reaction. Genomics 4: 198205

Kikuchi M, Hanamizu H, Narisawa K, Tada K (1989) Assay of methylmalonyl CoA mutase with high-performance liquid chromatography. Clin Chim Acta 184: 307-314

Ledley FD, Rosenblatt DS (1997) Mutations in mut methylmalonic acidemia: clinical and enzymatic correlations. Hum Mutat 9: 16

Ledley FD, Lumetta MR, Zoghbi HY, VanTuinen P, Ledbetter SA, Ledbetter DH (1988) Mapping of human methylmalonyl CoA mutase (MUT) locus on chromosome 6. Am J Hum Genet 42: 839846

Mancia F, Keep NH, Nakagawa A, Leadley PF, McSweeney S, Rasmussen B, Bösecke P, Diat O, Evans PR (1996) How coenzyme $\mathrm{B}_{12}$ radicals are generated: the crystal structure of methylmalonylcoenzyme A mutase at $2 \AA$ resolution. Structrue 4: 339-350

Nham SU, Wilkemeyer MF, Ledley FD (1990) Structure of the human methylmalonyl-CoA mutase (MUT) locus. Genomics 8: 710716

Niwa H, Yamamura K, Miyazaki J (1991) Efficient selection for highexpression transfectants with a novel eukaryotic expression vector. Gene 108: 193-199

Ogasawara M, Matsubara Y, Mikami H, Narisawa K (1994a) Molecular analysis of methylmalonic acidemia: identification of novel mutations in the methylmalonyl-CoA mutase gene with decreased level of mutant mRNA. Am J Hum Genet 55: A233 (abstract)

Ogasawara M, Matsubara Y, Mikami H, Narisawa K (1994b) Identification of two novel mutations in the methylmalonyl-CoA mutase gene with decreased levels of mutant mRNA in methylmalonic acidemia. Hum Mol Genet 3: 867-872

Sambrook J, Fritsch EF, Maniatis T (1989) Molecular cloning: a laboratory manual, 2nd edn. Cold Spring Harbor, New York

Thomä NH, Leadlay PF (1996) Homology modeling of human methylmalonyl-CoA mutase: a structural basis for point mutations causing methylmalonic acidemia. Protein Sci 5: 1922-1927

Toyo-Oka Y, Wada C, Ohnuki Y, Takada F and Ohtani H (1995) Molecular diagnosis of a kindred with novel mutation of methylmalonyl-CoA mutase gene using non-RI SSCP. Jpn J Clin Pathol 43: 625-629

Willard HF, Ambani LM, Hart AC, Mahoney MJ, Rosenberg LE (1976) Rapid prenatal and postnatal detection of inborn errors of propionate, methylmalonate, and cobalamin metabolism: a sensitive assay using cultured cells. Hum Genet 34: 277-283

Winship PR (1989) An improved method for directly sequencing PCRamplified material using dimethyl sulphoxide. Nucl Acids Res 17: 1266 